\title{
A Comparison of the Conductor Requirements for Energy Storage Devices Made With Ideal Coil Geometries*
}

\author{
W. Hassenzahl \\ Lawrence Berkeley Laboratory \\ University of California \\ Berkeley, CA 94720
}

August 22, 1988

Presented at the 1988 Applied Superconductivity Conference, San Francisco, CA, August 21-25, 1988

\footnotetext{
*This work was supported by the Director, Office of Energy Research, Office of High Energy and Nuclear Physics, High Energy Physics Division, U.S. Dept. of Energy, under Contract No. DE-AC03-76F00098 and by the Electric Power Research Institute.
} 


\title{
A COMPARISON OF THE CONDUCTOR REQUIREMENTS FOR ENERGY STORAGE DEVICES MADE WTTH IDEAL COIL GEOMETRIES*
}

\author{
W. Hassenzahl \\ Lawrence Berkeley Laboratory \\ University of California \\ Berkeley, CA 94720
}

\begin{abstract}
Superconducting Magnetic Energy Storage (SMES) plants have been proposed in both solenoidal and toroidal geometries. The former is efficient in terms of the quantity of superconductor required per unit of stored energy. For applicacions where a fringe field could be a problem, the toroidal geometry, which requires at least a factor of two more material, has been proposed. In addition to the solenoid and toroid, other geometries are possible, such as linear multipoles and spherical coils. These geometries have been considered for use in applications other than energy storage. In this report, the effectiveness (quantity of superconductor/stored energy) is calculated for various coil geometries.
\end{abstract}

\section{Introduction}

This report provides a comparison of various coil geometries in terms of the quantity of conductor, Qsc, required to store a given quantity of energy, E. In an earlier study, it was shown that a toroidal coil is less effective than a solenoid. ${ }^{1}$ Other coil geometries, such as long dipoles of the type used for accelerators and spherical coils considered were not included in these comparisons.

The effectiveness of the coils in storing energy could be expressed in several ways. For this evaluation, we choose to search for a maximum in the quantity $E / Q_{s c}$, where $E$ is in Joules, and $Q_{s c}$ is in Ampere-meters. The "maximum" is found by varying coil parameters, such as the ratio of diameter to length in a dipole.

It has been shown ${ }^{2}$ that the conductor requirements for coils depend on the geometry, the peak field, and the stored energy at the peak field level. In particular, for a given coil geometry, a large coil is more effective than a smaller one, and a coil producing a high field is more effective than one producing a lower field. The relationship is given by

$$
\mathrm{K}_{\mathrm{E}, \mathrm{B}, \mathrm{g}}=\mathrm{E} / \mathrm{Q}_{\mathrm{sc}}=\mathrm{C}_{\mathrm{g}}{ }^{-1} \mathrm{E}^{1 / 3} \mathrm{~B}^{1 / 3},
$$

where $\mathrm{C}_{\mathrm{g}}$ depends only on the coil geometry.
The quantities of interest in comparing various coils are $C_{g}$, which has units $\left(\mathrm{J}^{-2 / 3} \mathrm{~T}^{1 / 3} \mathrm{Am}\right)$ and, for a given field and stored energy, $\mathrm{K}_{\mathrm{g}}$ in (J/Am). The value $\mathrm{C}_{\mathrm{g}}$ can be used to determine the mass of superconductor for a given coil

$$
\mathrm{M}_{\mathrm{SC}}=\mathrm{C} \rho \mathrm{E}^{2 / 3} \mathrm{~B}^{-1 / 3} \mathrm{~J}^{-1},
$$

where $\rho$ is the density of superconductor in $\mathrm{kg} / \mathrm{m}^{3}$ and $\mathrm{J}$ is the average current density in the coil.

The analysis here will be valid for any type of conductor and any application. However, to make a fair comparison, both size and field must be fixed, thus the proposed Superconducting Magnetic Energy Storage (SMES) plants, having capacities of $5500 \mathrm{MWhr}$ and peak fields of about $5 \mathrm{~T}$, will be used as a reference point. Comparisons will be made on the basis of $\mathrm{C}_{\mathrm{g}}$ and $\mathrm{K}_{5,5, \mathrm{~g}}$, which are related by

$$
\begin{aligned}
\mathrm{K}_{5,5, \mathrm{~g}} & =\mathrm{C}_{\mathrm{g}}{ }^{-1}(5500 \mathrm{MWh})^{1 / 3}(5 \mathrm{~T})^{1 / 3} \\
& =4.64 \times 10^{4} \mathrm{C}_{\mathrm{g}}^{-1} .
\end{aligned}
$$

In the following sections, the notation $\mathrm{K}_{5,5, \mathrm{~g}}$ will be shortened to $\mathrm{K}_{\mathrm{g}}$. These calculations are for very large coils at a field of 5.0T, which implies that the thickness of the superconductor in the windings, only a few millimeters, is quite small compared to the other dimensions. This would not be true at a few tens of MJ of stored energy, where the optimization might be different.

The analysis below shows that the most effective coil for energy storage is the thin walled solenoid, which is the type of coil that is proposed for the SMIES program. The only other coil types that approach the solenoid in effectiveness are those that are topologically similar, and then only in the limit where they are geometrically similar.

\section{$\underline{\text { Toroids }}$}

Though the thin walled solenoid may be the simplest

*This work was supported by the Director, Office of Energy Research, Office of High Energy and Nuclear Physics, High Energy Physics Division, U.S. Dept. of Energy, under Contract No. DE-AC03-76SF00098, and by the Electric Power Research Institute.

Manuscript received August 22, 1988. 
coil to consider, the poloidal coil (subscript p)producing a toroidal field can be calculated in closed form and is considered first to show the approach.

This coil may be thought of as a winding that is formed on the surface of a donut with the conductor turns wound on the minor radius. One characteristic of this type of coil is the very low fringe field compared to the solenoid.

The inductance of a toroidal coil is given by ${ }^{3}$

$$
L=\mu_{0} R\left(1-\sqrt{1-b^{2}}\right)
$$

where $b$ is the ratio of minor radius, a, to the major radius $\mathrm{R}$. For a total current, $\mathrm{I}_{\mathrm{t}}$, the stored energy is

$$
E=\left(\mu_{0} R / 2\right)\left(1-\sqrt{1-b^{2}}\right) I_{t}^{2},
$$

the maximum field is

$$
B_{m}=\mu_{0} I_{l} / 2 R(1-b) \pi .
$$

and the quantity of conductor required for the coil is

$$
\mathrm{Q}_{\mathrm{SC}}=2 \pi \mathrm{aI}_{\mathrm{t}}=2 \pi \mathrm{RbI_{ \textrm {t } }} \text {, }
$$

which can be expressed in terms of the energy and field

$$
\begin{gathered}
Q_{s c}=2 \pi R \mathrm{bI}_{t}{ }^{4 / 3} / \mathrm{I}_{\mathrm{t}}^{1 / 3} \\
=\left(16 \pi^{2} \mathrm{~b}^{3} /\left(\mu_{\mathrm{o}}(1-\mathrm{b})\left(1-\sqrt{\left.1-\mathrm{b}^{2}\right)^{2}}\right)\right)^{1 / 3} \mathrm{E}^{2 / 3} \mathrm{~B}^{-1 / 3} .\right. \\
=C_{\mathrm{p}} \text { (b) } \mathrm{E}^{2 / 3} \mathrm{~B}^{-1 / 3} .
\end{gathered}
$$

Solving for $K=E / Q$,

$K_{p}=\left(\mu_{0}(1-b)\left(1-\sqrt{1-b^{2}}\right)^{2} 16 \pi^{2} b^{3}\right)^{1 / 3} E^{1 / 3} B^{1 / 3}$.

The values of $E / Q_{s c}$ are given in Table I for $b$ in the range 0.05 to 0.8 . Coils having smaller aspect ratios could be used, but they are even less effective in their use of conductor.

Table I. Stored energy per unit volume of conductor for a $5500 \mathrm{MWhr} 5 \mathrm{~T}$ toroidal coil.

$\begin{array}{lrrrrrr}\mathrm{b} & 0.05 & 0.1 & 0.2 & 0.5 & 0.6 & 0.8 \\ \mathrm{C}_{\mathrm{p}} & 2200 & 1770 & 1450 & 1320 & 1190 & 1200 \\ \mathrm{~K}_{\mathrm{p}} & 21 & 26 & 32 & 38 & 39 & 36\end{array}$

\section{Solenoids}

The simple, thin-walled solenoid with height $\mathrm{h}$ and radius $R$, which is described by the aspect ratio, $\beta=h / 2 R$, has been shown to be an effective geometry for energy storage. Most SMES. studies have developed coils with this geometry. The superconductor required in a solenoid is given by ${ }^{4}$

$$
Q_{S C}=C_{S}(\beta) E^{2 / 3} B^{-1 / 3},
$$

where the subscript $s$ refers to the solenoid. The inductance for the solenoid of rectangular cross section cannot be given in closed form; it must be calculated numerically. The general expression for the inductance of this type of coil was developed by Nagaoka ${ }^{5}$, but the usual reference, which has extensive tables, is the book Inductance Calculations by Grover. ${ }^{6}$ At present, a variety of computer programs can be used to calculate the inductance of a solenoid of almost any shape.

The stored energy per unit of conductor

$$
K_{S}=E / Q_{S C}=C_{S}(\beta)^{-1} E^{1 / 3} B^{1 / 3} .
$$

is calculated numerically and the results are given in Table II for aspect ratios between 0.01 and 1.0.

The variation of the effectiveness of solenoids with different aspect ratios, in terms of conductor utilization is quite evident. The "best" solenoid can store 83 Joules for each Ampere-meter of conductor, whereas the lowaspect-ratio, which is preferred for SMES due to its low outward radial pressure, coil stores only 55.

Another type of solenoidal coil, one having a circular cross section, the poloidal coil, which carries a toroidal current, is also a relatively effective coil in terms of $E / Q_{s c}$. At an aspect ratio of 0.1 , it stores $74 \mathrm{~J} / \mathrm{Am}$. In the limit of very low aspect racios, it approaches the solenoid in terms of effectiveness. The values of $C_{t}$ and $\mathrm{K}_{\mathrm{t}}$ for the poloidal winding are also given in Table II.

Table II. The effectiveness of thin-walled solenoids of different aspect ratios.

\begin{tabular}{lcccccr}
$\beta$ & 0.01 & 0.05 & 0.1 & 0.2 & 0.5 & 0.8 \\
$\mathrm{C}_{\mathrm{s}}$ & 800 & 620 & 580 & 560 & 600 & 640 \\
$\mathrm{~K}_{\mathrm{S}}$ & 55 & 74 & 80 & 83 & 77 & 71 \\
$\mathrm{C}_{\mathrm{t}}$ & 830 & 650 & 630 & 650 & 940 & 2050 \\
$\mathrm{~K}_{\mathrm{t}}$ & 56 & 71 & 74 & 71 & 57 & 23 \\
& & \multicolumn{4}{c}{ Linear, Axial Coils }
\end{tabular}

From time to time, the concept of a long axial 
current with some sort of return path is proposed for an energy storage device. This case is usually a part of a force-reduced coil geomerry, which has been shown not to be valid. ${ }^{7}$ The axial current above needs a return path. When this return path is included, the axial current can be dismissed quickly as a separate geometry because it can be considered as either a toroid or a solenoid, depending on the form of the return path. In either case, the shape is at an extreme limit of aspect ratios, $b$ or $\beta$, and thus, it is ineffective in the use of superconductor.

\section{Linear Multipole Coils}

Another group of coils that are frequently used, though not generally considered for energy storage, are the linear multipole coils. These coils are characterized by having a long axial region in which the field pattern does not vary. They are used in accelerators for bending and focusing beams of charged particles.

Because these coils have a "two-dimensional" field over a long region, they do not follow the same scaling law as the solenoid and the torus, though there is a limit, discussed below, where the dipole approaches the solenoidal form.

The fields in the linear multipole are most simply expressed in cylindrical coordinates. The current, which is axial, may be thought of as a sheet on the surface of a cylinder. The circumferential distribution of current is sinusoidal and is given by

$$
J=J(\theta) z=J_{Z} \cos (n \theta) \delta(r-R) z,
$$

where the bold print indicates a vector quantity.

The value of $\mathrm{n}$ determines the multipole number, the actual number of poles being $2 n$. The delta function places the current sheet at a radius $R$.

This current produces a vector potential $A=A_{z} z$. Where $A_{z}$ is of the form

$$
\begin{array}{ll}
A_{z}=\frac{\mu_{0} J r^{n}}{2 n R^{n-1}} \cos (n \theta) & r<R \\
A_{z}=\frac{\mu_{0} J R^{n-1}}{2 n r^{n}} \cos (n \theta) & r>R
\end{array}
$$

The fields inside and outside appear to be two dimensional in the approximation that the coil is very long. In fact, this approximation is not valid for short coils and a correction is needed for the ends. We start with a calculation of stored energy for the two dimensional case; then a correction is given for the end effects in the form of added conductor for the return path.

The stored energy per unit length is

$$
\begin{aligned}
E_{\text {in }} & =\int_{0}^{R} r d r \int_{0}^{2 \pi} d \theta B^{2} / 2 \mu_{0} \\
& =\pi \mu_{0} R^{2} J^{2} / 8 n . \\
E_{\text {out }} & =\int_{R}^{\infty} r d r \int_{0}^{2 \pi} d \theta B^{2} / 2 \mu_{0} \\
& =\pi \mu_{0} R^{2} J^{2} / 8 n .
\end{aligned}
$$

The total energy in a length $L$ is thus

$$
\begin{aligned}
E & =\pi \mu_{0} R^{2} J^{2} \ell / 4 n=\pi R^{2} B^{2} l / \mu_{0} n \\
& =\frac{2}{n}\left(\pi R^{2}\right)\left(B^{2} / 2 \mu_{0}\right) l .
\end{aligned}
$$
coil is

The total superconductor requirement for this type of

$$
\begin{aligned}
\text { Qsc } & =4 \pi \int_{0}^{\infty} r d r \int_{0}^{2 n} d \theta J \cos (\theta) \delta(r-R) l \\
& =4 R J \ell
\end{aligned}
$$

The effectiveness of this coil is then

$$
\mathrm{K}_{\mathrm{d}}=\mathrm{E} / \mathrm{Q}_{\mathrm{sc}}=\pi \mu_{\mathrm{o}} \mathrm{RJ} / 16 \mathrm{n}=\pi \mathrm{R} \mathrm{B} / 8 \mathrm{n} .
$$

This expression shows that of the linear multipoles, only the dipole, where $n=1$, need be considered because all higher multipoles are less effective by a factor $1 / n$.

The effect of the end of the coil is to increase the conductor requirements without significantly changing the stored energy. The increase is due to the additional material required at the end for the current to turn around. The quantity of superconductor required in a real coil can be approximated by

$$
\mathrm{Q}_{s c}=8 \mathrm{BR}(\ell+2 \mathrm{R}) / \mu_{0} \text {, }
$$


and the effectiveness of the coil becomes

$$
K_{d}^{\prime}=(\pi B R / 8 n)\left(/ / L+2 R^{\prime}\right)
$$

The quantity $R^{\prime}$, which is usually somewhat larger than $R$, is the length of the return path that the conductor must make at the ends of the coil. In most applications, the length is much greater than the radius so the value in parentheses, $\ell+2 R '$, reduces to the length. For short coils, where $l=R$, the above equation for stored energy is no longer valid. And, for $n=1$, the coil begins to look like a solenoid.

The values of $\mathrm{K}_{\mathrm{d}}$ are given in Table III for dipoles of different lengths and radii, and the effect of the ends is apparent.

Table III. The dimensions and the effectiveness, in terms of superconductor requirements, for several $5500 \mathrm{MWhr}, 5 \mathrm{~T}$ dipole coils.

$\begin{array}{lrrrrrr}R(m) & 10 & 20 & 30 & 40 & 50 & 70 \\ \ell(m) & 3200 & 800 & 356 & 200 & 128 & 65 \\ K_{d}{ }^{a} & 20 & 39 & 59 & 79 & 98 & 137 \\ K_{d} b^{b} & 20 & 38 & 53 & 63 & 66 & 60\end{array}$

a. Effectiveness in the 2-d, approximation.

b. Effectiveness with a realistic end approximation.

\section{Spherical Coils}

This class of coils is restricted to circular, poloidal, currents on the surface of a sphere, though more complicated current shapes are possible. Spherical polar coordinates are used to describe the magnetic fields and the currents for coils of this geometry. The most effective current distribution is given by the expression

$$
\mathrm{J}=\mathrm{J}_{\mathrm{O}} \emptyset=\sum_{\mathrm{m}, \mathrm{n}=0}^{\infty}\left(\mathrm{I}_{\mathrm{n}} \sin (\mathrm{n} \theta)+\mathrm{I}_{\mathrm{m}} \cos (\mathrm{m} \theta)\right) \emptyset .
$$

In this coordinate system, the fields are pure multipoles when the values of $\mathrm{I}_{\mathrm{n}}$ and $\mathrm{I}_{\mathrm{m}}$ are related as coefficients of the Legendre polynomials. The expressions for the stored energy and the quantity of conductor required are more complicated than for the earlier cases. However, as for the linear-multipole coils, the higher multipoles are less effective than the lower order terms. Thus, we need only consider the analog to the dipole. In this geometry there are two dipole forms. The simplest to analyze, and the most effective, is the case where $I(\theta)=I_{1} \sin \theta$.
For this case, after a bit of calculation, the effectiveness of the coil is found to be $\mathrm{K}_{5,5, \mathrm{sp}}=72$ $\mathrm{J} / \mathrm{Am}$, which approaches the effectiveness of the solenoid.

\section{Conclusion}

The "best" coil for energy storage is obviously the thin-walled solenoid, as shown below in Table IV.

Table IV. Relative effectiveness of different coil geometries for a $5500 \mathrm{MWhr}, 5 \mathrm{~T}$ SMES unit in terms of Joules/Ampere-meter.
Coil description

Thin-walled solenoid "best"

Thin-walled solenoid LAR ${ }^{a}$

Poloidal coil

Linear dipole

Spherical dipole

Toroidal coil

a Low Aspect Ratio
Relative Effectiveness

83

55

74

66

72

39

\section{References}

1. W. V. Hassenzahl, editor, "Progress Report on the Superconducting Magnetic Energy Storage Program," Los Alamos Scientific Laboratory Report, LA-5472PR (December 1973).

2. Wisconsin Superconductive Energy Storage Project, Vol. 1, University of Wisconsin Publication (1974).

3. Z. J. J. Stekly, "Magnetic Energy Storage Using Superconducting Coils," AVCO Everitt Research Laboratory Report, AMP-102, and Proc. IDA Pulsed Power Conf., Vol. 1, 63-142 (March 1963).

4. Rabinowitz suggested that for very small $\beta$ the quantity of superconductor will be proportional to $E$, not to $E^{2 / 3}$.

5. Nagaoka, Journal of the College of Science, 27, 18, Tokyo, Japan (1909).

6. F. W. Grover, "Inductance Calculations: Working Formulas and Tables," Dover Publications, Inc., New York, NY (1962).

7. W. V. Hassenzahl, "Considerations Against A Force Compensated Coil," LBL-24993, elsewhere in these proceedings. 